somewhat thickened at the tip (thus approaching club wheat in form) and a number of others having long narrow heads with the spikelets set very far apart, almost exactly like true spelt. In most of the latter the kernels are very firmly held by the chaff.

The cross made between Red Fife and Polish wheat gave only one seed, and progeny have been retained almost in full, up to the present time. The same tendency to vary in the third generation is observed here, and few, if any of the types, can be regarded as fixed. The total number produced so far is nearly forty. These include not only a large number of forms intermediate in character between the two parents but also some which present peculiarities not found in either. For instance there are observed short compact neads, and long heads of the spelt type, while several specimens have downy chaff, the chaff of both of the parents being smooth.

The crosses made between Red Fife and two varieties of Triticum durum have also given some extremely interesting results, but the study of last season's harvest could not be completed in time to present the facts in this paper. Some remarkably vigorous types have been obtained which it is hoped may be of permanent value.

Perhaps the most important lessons enforced by the foregoing observations are these: That it is extremely unsafe in the present state of our knowledge of the subject to make prognostications in regard to the breeding of wheat; that fixed types are not often obtained in less than three or more years, even when the seed from each plant is sown separately each year; and that, for the production of a varied assortment of types, a simple cross between two distinct varieties is all that is necessary; the use of parents known to be of complex descent not being an essential feature as is sometimes maintained.

An extensive series of experiments was started by the writer last season in which the same cross was made in opposite ways, and it is hoped that these tests will give much further information in regard to the relative influence of the male and female in the breeding of wheat.

\title{
NOTES ON CITRUS HYBRIDS.*
}

By Herbert J. WEBber, Physiologist, In Charge of Plant Breeding Laboratory, U. S. Department of Agriculture.

\section{OBJECTS OF THE WORK.}

Several years ago experiments were started by the Department of Agriculture in the breeding and improvement of citrus fruits. These experiments were at first carried on jointly by the writer and $\mathrm{Mr}$. W. T. Swingle, also of the Department of Agriculture. Since

*While the substance of this paper was presented at the meeting of the American Breeders' Association at St. Louis, December, 1903, it has been revised and brought up to date for publication, so that data derived since the presentation of the paper has been included.-EDITOR. 
the making of the crosses, however, the work has been in charge of the writer. The most important improvements sought were to secure (1) more hardy or frost-proof varieties; (2) loose-rind, kidglove types of the common orange; (3) loose-rind types of the pomelo; (4) new fruits intermediate in quality between the orange and pomelo, having the sprightly acid flavor of the pomelo, but with the bitter principle reduced and in general sweeter than the pomelo. Various hybrids were made having other objects in view, but the foregoing were the principal objects sought.

\section{SUMMARY OF RESULTS ACCOMPLISHED.}

A large number of the hybrids have now fruited, and several striking new fruits have been obtained. A new group of hardy fruits has been originated which are intermediate between the two parents, that is, the hardy Citrus trifoliata and the tender C. aurantium. This new group differs from any other known group of citrus fruits, and has been termed the citrange. A second new group has been obtained by hybridizing the tangerine and pomelo, and this group, which is strictly intermediate between the two parents, has been termed the tangelo. The following is a list of the new fruits which have been produced:

(1) Rusk citrange, a cross between the orange (female parent) with the trifoliate orange (male parent). This fruit is smooth, nearly round, is about $1 \frac{7}{8}$ to 2 inches in diameter, and is very juicy and nearly seedless.

(2) Willits citrange, a cross of the trifoliate orange (female parent) with the common orange (male parent). The fruit is nearly round, is about 2 or $2 \frac{1}{2}$ inches in diameter, is very juicy and nearly seedless, and is rough and ridged on the surface.

(3) Unnamed citrange, a hybrid between trifoliate orange (female parent) with common orange (male parent). The fruit is fully as large as the common orange and in general of the same appearance. It measures 3 inches or more in diameter, has the color of the common orange, and has slight longitudinal grooves which clearly distinguish it. All of the fruits of this variety thus far tested are totally seedless.

(4) Sampson tangelo, a hybrid between pomelo (female parent) and Dancy tangerine (male parent). The fruit is about 2 or 33 inches in diameter, the appearance is very attractive, and the flavor is sprightly acid and slightly bitter. The distinct flavor coupled with the character of loose rind, bids fair to make it a valuable fruit.

(5) Unnamed tangelo, a cross of pomelo (female parent) with Dancy tangerine (male parent). In general this seedling has the characters of the Sampson tangelo, but the fruit is much larger and differs in shape, being pyriform, and is from 3 to $3 \frac{1}{2}$ inches in diameter.

(6) Weshart tangerine, a cross of Dancy tangerine (female parent) with Parson Brown orange (male parent). While this was a careful hand-made hybrid, the fruit has all the characters of the best tangerine but is considerably larger and ripens about two weeks earlier.

(7) Trimble tangerine, a cross of the Dancy tangerine (female parent) with Parson Brown orange (male parent). This fruit has the same characters as the Weshart, but is if anything slightly larger and more highly colored, and is possibly not quite so highly flavored as the Weshart.

(8) Unnamed hybrid between the sweet orange (female parent) and pomelo (male parent). This fruit is simply a good highly flavored ordinary orange having but few seeds and showing streaks of blood, a character which was not 
visible in either of the parents. While it is totally different from the orange used as the female parent, it shows no characters which seem to have been derived from the pomelo. (Pls. II and III, No. 970.)

The above are the only varieties which thus far are known to possess value. A number of others, however, appear from preliminary tests to be promising, and other new varieties will doubtless be produced in the course of the experiments. It is not the purpose of this paper to describe these fruits but to call attention to some interesting features which have been brought out in the course of the experiments.

\section{COMPARISON OF HYBRIDIZATION WITH SELECTION.}

The experiments furnish an interesting illustration of the results obtained by hybridization as compared with those obtained by selection in the development of new varieties. Ever since the orange industry was established in this country, more or less severe freezes have occurred at intervals, showing the necessity of securing hardy varieties. These conditions resulted in growers making observations and selections of apparently hardy seedlings. All over Florida the sweet seedling orange has been in great favor until recently, and very many groves even now are simply sweet seedlings of unnamed varieties. The various freezes which have occurred have thus always given an opportunity for a test on a large scale of the hardiness of sweet seedling oranges, and any striking variation toward hardiness would be immediately noticed. We thus have an experiment on a grand scale in the production of a hardy orange by seed selection, as several million seedlings have doubtless come under the careful observation of growers. While many varieties have been selected out and claimed to be hardy, nevertheless none of them have proven to be more hardy in any marked degree than the ordinary orange. The hope, therefore, of securing markedly hardy varieties by the selection of the pure sweet orange is very remote. By the use of hybridization, on the contrary, we have been able to make striking advance in a very few years. The trifoliate orange is a wild type, worthless so far as edible qualities are concerned, which is grown as a hedge plant as far north as New York and Philadelphia. By hybridizing this hardy orange with the sweet orange it has been possible to produce a new group of fruits, the citranges, which are intermediate in the character of hardiness, and the resulting fruit is an edible orange of distinct value which can be grown from 300 to 400 miles north of the present orange-growing belt. It is doubtful whether it would ever have been possible to secure an edible citrus fruit of the same hardiness by means of selection. The most promising method for the production of such a hardy edible fruit by means of selection would doubtless have been to select the trifoliate orange for quality of fruit rather than to select the common orange for increased hardiness. No experiments have been carried on in the selection of the trifoliate orange, but there is certainly con- 
siderable variation in the quality and shape of the fruits produced by different trifoliate seedlings, so that a continuous selection through a number of generations would doubtless result in the production of a much improved quality of fruit combined with a reduction in the number in seeds.

\section{USE OF CLONAL TYPES.}

A factor of importance in plant breeding is the difference between clons and seed races. Clons, or clonal varieties, it will be remembered are those varieties of plants which are propagated entirely by vegetative parts, and are thus reproduced parts of the same seedling. Clonal varieties thus include those sorts propagated by buds, grafts, cutting, tubers, slips, runners, suckers, etc. In the breeding of clonal varieties like the orange any seedling obtained which possesses desirable characters can be propagated by budding and is thus of immediate value. In the case of plants propagated by seed, such as wheat, corn, oats, cotton, etc., a hybrid may be secured in possibly the second or third generation which has exactly the qualities that are desired in the new variety. However, even when the hybrid is once found it is by no means certain that it will be possible to fix it and render it true to type. The process in such cases is to inbreed the hybrid with its own pollen or with pollen of a similar hybrid and reject the progeny which do not reproduce the desired qualities. Experience has shown that in some cases it is possible to gradually breed a hybrid to a state of fixity by'this method, but in many other cases it has been found impossible to fix a hybrid of the type desired even when jt has once been developed. Thus, while in the case of fruit trees a long period must elapse between the time of making the cross and fruiting the plant, yet the process is quicker and shorter than in the case of breeding racial varieties, owing to the fact that we can propagate a hybrid when once secured by budding or grafting.

\section{VARIATION IN THE FIRST GENERATION.}

The recent literature bearing on Mendel's law emphasizes the fact that hybrids in the first generation usually come nearly alike in almost all characters. While this may be the general rule in the case of hybrids of racial varieties, it certainly is not true in the case of hybrids of clonal varieties where neither parent is fixed or propagates true to seed. In the case of hybrids of the trifoliate orange (female parent) with the common orange (male parent), both parents were probably as nearly fixed in type as ordinary seed races, although they doubtless would have shown some slight individual variations. The seedlings of the trifoliate orange in general are as nearly alike as different corn or wheat plants, and ordinary sweet orange seedlings are usually as nearly alike as different corn or wheat plants, so that in this case two different species were crossed both of which would practically have come true to seed. Nevertheless the seedlings of the first generation were found to be very variable. Indeed no two seedlings have thus 
far fruited which are not strikingly distinct. Only a few of these hybrids have thus far fruited, but in every case the fruits are sufficiently different to be distinguishable by a novice, being different in size, shape, color, appearance, quality, texture, etc. The trees of all of them also are very different in foliage and branching. All hybrids of the above combination have trifoliate leaves which are intermediate between the two parents. The leaves are mainly trifoliolate with a few unifoliolate, and these are uniformly much larger than those of the trifoliate orange and of slightly different texture, showing plainly the intermediate texture. Some of the seedlinge have a strikingly distinct character of branching differing from either parent type. Some of them have very compact foliage, similar to the boxwood tree, and give evidence of being of value for hedge plants. Others however, have comparatively open foliage nearly like the ordinary trifoliate orange, but with the branches in general ascending. In still other cases the limbs show a decided tendency to droop and bend downward, forming low and spreading instead of upright plants.

\section{POLYEMBRYONIC VARIATIONS.}

An important feature connected with citrus hybridization is the fact that throughout the genus, the phenomenon of polyembryony is common, that is, several embryos are frequently developed from a single seed. So far as this is understood at present it would seem that one embryo develops from the egg cell in the normal way after fecundation. This seems to stimulate to development certain cells of the nucellus of the ovary which lie outside of the embryo sac. These cells grow, divide, and finally form embryos which push into the embryo sac, resulting in embryos of almost the same form as that developed from the egg cell proper. It should be added, however, that so far as the process of fecundation is understood at the present time, these abnormal embryos develop directly from the cells of the mother tissue without definite indication of any effect of the male element other than possibly a slight stimulation or irritation due to the incipient development of the fecundated egg cell. It would thus be expected that any seedling grown from these abnormal embryos would have the same character of plant and fruit as the mother parent. They should be as nearly alike as trees produced from different buds of the Washington Navel orange or of the Baldwin apple. This, however, as will be shown later, is not the case. It is clear from the results of experiments already completed that there is a striking difference between the hybrid seedling developed from the egg cell proper and those developed from these adventive embryos. In crossing the Trifoliate orange with the common orange, it is not infrequent to find two or three seedlings developed from the same seed. In this case the seedling which resulted from the hybridization can clearly be distinguished by the larger leaves showing the common parentage. The same effect is brought out much more clearly in cases where the common orange is crossed with pollen of the trifoliate orange. Here all of the seedlings which develop from these 
polyembryonic embryos have the unifoliolate leaves of the common orange, while the seedling developed from the egg cell proper which was hybridized with the trifoliate orange is clearly distinguishable by having trifoliolate leaves. In further experiments with trifoliate seedlings this fact is still further brought out, as in all cases the intermediate hybrids which were thought to have developed from the egg cell as a result of hybridization clearly showed the influence of the male parent in fruit and tree characters, while the other seedlings supposed to have developed from the polyembryonic embryos in no case have showed any influence of the male parent. The same is true in cases of hybrids between the pomelo (female) and Dancy tangerine (male). For instance, a pomelo which had been hybridized with the Dancy tangerine produced in all 76 seeds which when grown gave 106 seedlings. A number of the seeds failed to germinate, so that very many of the seeds produced more than one seedling. Only 5 of these 106 seedlings showed any influence of the Dancy tangerine parentage in the foliage characters. All of the other seedlings seemed to be pure pomelo. One of these 5 intermediate seedlings has fruited and this is the Sampson tangelo mentioned above, which is clearly an intermediate hybrid. Of the other 101 seedlings about 20 have produced fruit and in every case these fruits are apparently pure pomelo. These have shown no characters of the tangerine so far as can be distinguished.

From the above illustrations it is clear that two types of seedlings commonly result in the hybridization of oranges: One type which shows true hybridization, and another, presumably developed from these polyembryonic embryos, which are apparently not hybrids in any sense of the word, and would be expected to come true to the type of the mother parent and as uniform as trees propagated from buds of, the same variety. The most interesting fact, however, is that the seedlings, which without question developed from these polyembryonic or adventitious embryos, show new characters and variations far in excess of what would be expected in the production of a clonal variety. It is difficult to understand the cause of this variation, unless some influence of the male parent is carried over, or some reason for the variation exists which is not now understood. It is therefore thought justifiable to speak of this variation as a new type of variation. The following illustrations will show what striking variations occur in this direction:

In the case of crosses between the Dancy tangerine with pollen of the Parson Brown orange, all of the true hybrids would certainly be expected to show characters in the fruit and foliage serving to distinguish them from the orange or tangerine. Several seedlings of this cross, however, have fruited, and none of them show foliage or fruit characters which indicate the orange parentage. Yet these seedlings differ markedly from the Dancy tangerine which was used as the mother parent. The fruits are all true tangerine in every character but they are very much larger and earlier in general than the Dancy, the mother parent, and are as a whole of slightly better quality and would never be confused with that variety. Two of them 
have been thought sufficiently different from the Dancy tangerine to be named as new varieties. These are the Weshart and Trimble tangerines mentioned above.

Another illustration is found in the crosses of the common orange with the pomelo (shown in Plates II and III). The foliage characters of this series indicate nothing but pure orange and the same is true of the fruit characters. In no character do they show pomelo parentage. Yet the seedlings differ among themselves strikingly, and it is possible to obtain a number of new clonal types from the seedlings derived from this one series all taken from a single hybrid fruit. In one of the seedlings shown (No. 966, Pls. II and III) the rind is smooth, glossy, and very thin, and the seeds are abundant. In the second fruit (No. 970) which is nearly seedless, the rind is rough, with little depressions over the large oil glands, and thicker than in the preceding case. If the fruits of these two seedlings were mixed any inexperienced person would have no trouble in separating them. Such a separation of fruits, as anyone who is familiar with orange varieties knows, is impossible with very many of the varieties of the orange. Very few persons indeed can tell the fruits of different orange varieties apart after they are mixed. There is little question that these two seedlings were developed from polyembryonic or adventive embryos, as they show no effect of hybridization.

Another striking illustration of the same sort is found in a series of hybrids of the pomelo and orange. All of the seedlings of this series which have thus far borne fruit show no indication of the orange parentage. They are all typical of the pomelo in shape, size, color, foliage, flavor, and all other characteristics. Yet of 20 seedlings of this series which have thus far fruited, 5 or 6 different varieties or clons could easily be distinguished. They differ in number of seeds, thickness and color of rind, and other details.

It would thus appear that these polyembryonic embryos in various citrus fruits give variations between themselves as great, if not greater, than embryos developed in the normal way from fecundated embryos where no hybridization has taken place. As stated above, if the origin of these embryos is understood correctly-and our knowledge of the subject is limited to the studies by Strasburgerthey are developed from certain cells of the mother tissue surrounding the embryo sac, and do not contain any of the male matter brought over in fecundation. Trees grown from these embryos would thus be similar to the reproduction of varieties by buds inasmuch as in each case the new tree comes from certain cells of the mother variety without the intervention of male matter, and we would normally expect such seedlings to be as similar to each other as trees from different buds of the same clon or varicty. The writer is unable to offer any definite explanation of this variation in seedlings from polyembryonic embryos, but would offer the following suggestions. The reason may be found in the irregular cell divisions of the nucellar tissue from which these embryos develop. The reducing division is supposed to take place in the division of the archegonial cell which first gives rise to four cells one of which becomes 

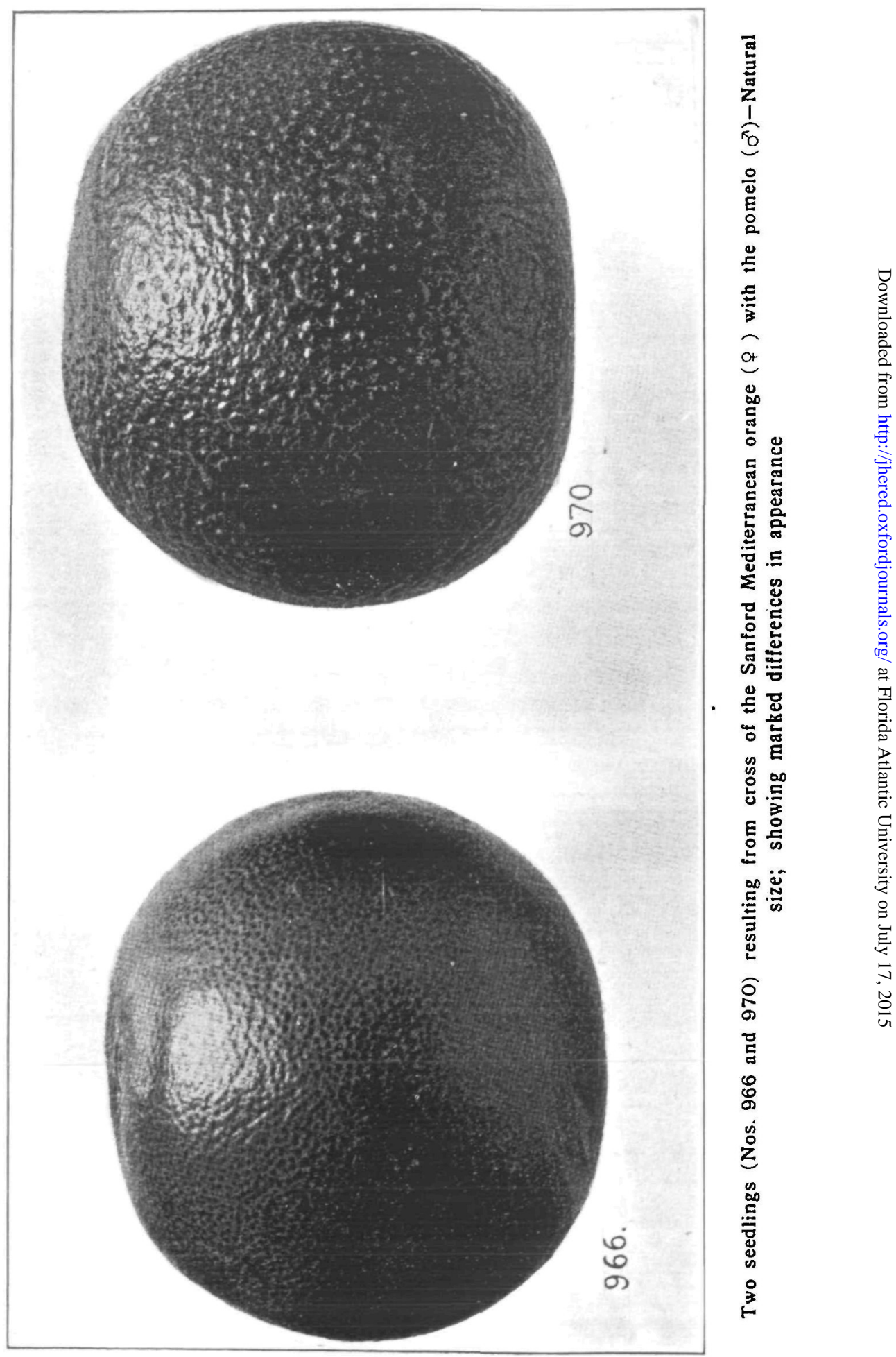


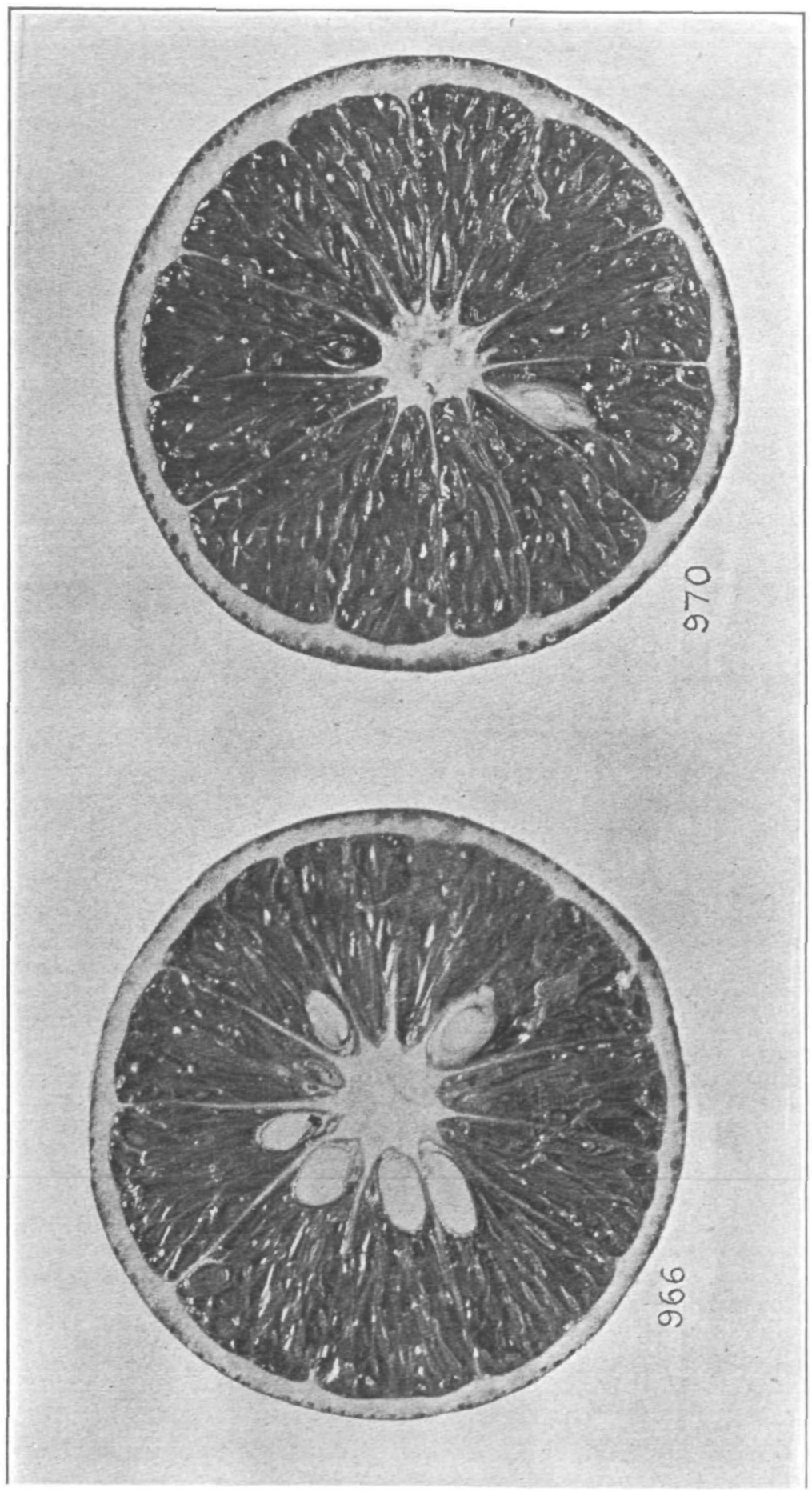

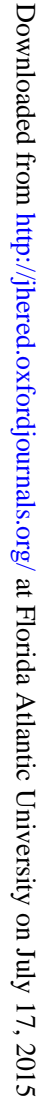


differentiated into the embryo sac. The other cells which do not become differentiated to form the embryo sac are supposed to have a reduced number of chromosomes and to be crowded to the wall and possibly absorbed during the development of the embryo sac. It might be possible that certain of these cells with reduced chromosomes unite with neighboring cells or possibly divide without such union and give rise to the several adventive embryos, thus in a measure accounting for the variation and segregation of the characters. On the other hand, it is known that the tissue of the interior of the nucellus of the ovary is largely of sporogenous nature and no noticeable differentiation exists at first between the archegonial cell which finally gives rise to the embryo sac and the surrounding cells. It may be possible that certain of the nucellar cells divide in such a way as to give a segregation of the characters and later give rise to the several embryos, and thus account for the variation. Again, we might assume that certain masses of the protoplasm may pass over from the male parent to these cells. In some of the cycads it is claimed by certain investigators that there is an intercommunication of the cytoplasm between the embryo sac and the surrounding cells, and that even solid masses like starch grains pass from the surrounding cells into the embryo sac. The recent investigations in fecundation have tended more and more to the belief that a complete cell, including the nucleus and cytoplasm, passes over in fecundation. The male nucleus with its cytoplasm would be expected to unite with the nucleus and cytoplasm of the egg cell proper. On the other hand, a certain amount of the cytoplasm of the pollen tube in many cases is known to pass into the embryo sac and in cases where double fecundation occurs we know that a second sperm nucleus passes down into the embryo sac and unites with the endosperm nucleus. So far as the writer is informed it has never been claimed that this second nucleus is accompanied by any material amount of cytoplasm and could in any sense be considered a complete cell although this matter is probably in doubt. It may be assumed from what is known of the process of fecundation in other plants, that in the fecundation of the orange at least some of the cytoplasm from the male parent passes over into the embryo sac, and further that certain particles of this cytoplasm could pass out into the cells of the nucellus adjoining the embryo sac if any communication existed. Such particles might be assumed to influence the developing cells and give rise to the variation. The writer is aware that cytoplasmic matter, according to our present understanding, would not be supposed to have any effect in influencing hereditary characters, but our understanding of this matter is far from perfect.

While the writer has made no statistical comparison of the amount of variation in seedling oranges-sothat it is impossible to make positive statements yet-he has made examinations of a very large number of seedling trees of oranges and pomelos which would be supposed to have come promiscuously from embryos fecundated in the normal way (not hybrids) and from adventive embryos. Among such seedling trees there does not appear to be nearly so great a 
variation between different individuals as occurs among different individuals developed from the polyembryonic seeds of the hybrids under discussion. The writer is thus forced to the conclusion that there must be some influence, either direct or indirect, of the male parent. It would seem that such influence is limited in some way to the imparting of a tendency to the segregation of characters already existing in the mother parent and not to the transmission of any of the characters of the male parent. Citrus fruits, it will be remembered, are a very highly developed class, the varieties being probably of very mixed origin. The evolution or amelioration has progressed so far in the ordinary orange that fruits may develop without pollination, as shown by the writer's experiments. In several instances fruits of the Navel orange have been developed in the course of the experiments where all pollination was precluded by emasculation and bagging. The same has been proven also to be the case with certain varieties of the ordinary sweet orange, as, for instance, the St. Michael, in which several experiments have led to the development of fruits where all pollination was precluded. In some of these cases, furthermore, seeds developed without pollination. In such cases it would seem that the tendency to form adventitious or polyembryonic embryos has become so strong that they occasionally develop where pollination does not take place and where there is thus no stimulation derived from a developing fecundated egg cell. The writer believes from the results of hybridization experiments which have come under his notice that in very many instances of hybridization where widely distinct parents are used, that a large number of seeds develop simply as a result of the formation of adventive embryos without the intervention of fecundation and true hybridization, although the stimulus given by the hybridization of few seeds and the growth of the pollen tubes doubtless has considerable influence in inducing the development of these adventitious embryos. It is very important that a further study of this question of polyembryonic variation be carried out in connection with special cytological studies to determine if possible the reason for the variation.

\section{THE STERILITY OF HYBRIDS.}

In all of the hybrids between the trifoliate orange and the common orange, regardless of which species was used as the female parent, the hybrids which show true intermediate nature, are nearly sterile so far as seed production is concerned, although many of them are very prolific in the formation of fruit. The Rusk citrange is very prolific in fruit formation but only about half of the fruits produce seeds. The Willits, while not as prolific as the Rusk, produces only about one seed to four fruits. Several of the other hybrids which have thus far not been named, are practically seedless, some of them not yet having produced a single seed. Crosses of other species of citrus, as, for instance, Citrus aurantium with C. decumana, C. aurantium with $C$. nobilis, and $C$. nobilis with $C$. decumana, are all so far as observed, perfectly fertile, producing a normal number of seeds. 